\title{
Absence of the G1528C (E474Q) Mutation in the $\alpha$-Subunit of the Mitochondrial Trifunctional Protein in Women with Acute Fatty Liver of Pregnancy
}

\author{
ANIRBAN MAITRA, RANA DOMIATI-SAAD, NICOLE YOST, GARY CUNNINGHAM, \\ BEVERLY BARTON ROGERS, AND MICHAEL J. BENNETT
}

Departments of Pathology [A.M., R.D-S., B.B.R., M.J.B.], Obstetrics and Gynecology [N.Y., G.C.], and Pediatrics [M.J.B.], University of Texas Southwestern Medical Center, Dallas, Texas 75390, U.S.A.

\begin{abstract}
ABS
Acute fatty liver of pregnancy (AFLP) is a rare and dreaded
complication of pregnancy, almost exclusively seen in the third
trimester. The histopathologic features of AFLP closely resemble
those seen in metabolic disorders characterized by deficiency of
fatty acid oxidative enzymes. Several reports have established a
strong association between AFLP in the mother and fetal defi-
ciency of the enzyme long-chain L-3-hydroxyacyl-CoA dehydro-
genase (LCHAD). However, these studies have an inevitable
selection bias resulting from ascertainment through an affected
infant, rather than an unselected population of patients with
AFLP. We retrospectively examined a series of 10 women with
pregnancies complicated by AFLP to determine the prevalence
of the common LCHAD mutation (G1528C) in this population.
The existing LCHAD primers, which produce a 640-bp amplicon
\end{abstract}
(IJlst L, Ruiter JP, Hoovers JM, Jakobs ME, Wanders RJ: J Clin Invest 98:1028-1033, 1996), were modified to make them amenable to analysis of fragmented DNA obtained from microdissected formalin-fixed material. None of the patients were found to harbor the common G1528C mutation. It is likely that AFLP arising in the context of fetal LCHAD deficiency represents only one of the possible etiologies for this uncommon disorder, and the metabolic basis of AFLP is more heterogeneous than previously believed. (Pediatr Res 51: 658-661, 2002)

\section{Abbreviations:}

AFLP, acute fatty liver of pregnancy

LCHAD, long-chain L-3-hydroxyacyl-CoA dehydrogenase HELLP, hemolysis, elevated liver enzymes, low platelets
A spectrum of maternal liver disorders can occur during pregnancy. Many, such as hepatitis or drug-induced hepatotoxicity, can also be seen in nonpregnant individuals, whereas others, including preeclampsia and intrahepatic cholestasis of pregnancy, are unique to the gravid state (1). AFLP is an uncommon but clinically severe form of hepatopathy that is seen almost exclusively in the third trimester of pregnancy. AFLP manifests with jaundice, which can rapidly progress to severe coagulopathy, fulminant hepatic failure, and death of both mother and fetus. Approximately $50 \%$ of affected patients have preeclampsia with high blood pressure, proteinuria, and peripheral edema, and up to $20 \%$ may have AFLP coexisting with the HELLP syndrome (1). AFLP derives its name from the presence of marked microvesicular steatosis in the liver, often with involvement of the heart, kidneys, and pancreas. Recent studies have reported a maternal mortality rate of

Received June 8, 2001; accepted November 9, 2001.

Correspondence and reprint requests: Michael J. Bennett, Ph.D., FRCPath., Department of Pathology, University of Texas Southwestern Medical Center, Children's Medical Center of Dallas, 1935 Motor Street, Dallas, TX 75235, U.S.A.; e-mail: Michaelj.Bennett@UTSouthwestern.edu
$10-20 \%$ with AFLP, underscoring the need for early diagnosis and intervention. The cornerstone of therapy is delivery of the fetus, and the clinical condition dramatically ameliorates in the postpartum period.

In recent years, investigators have speculated that AFLP/ HELLP syndrome and certain inherited fatty acid oxidation disorders, specifically LCHAD deficiency, may share a common pathogenic mechanism, based on striking similarities in hepatic pathology. This empirical association was strengthened by reports of AFLP or HELLP syndrome occurring during the third trimester in mothers bearing children with LCHAD deficiency. For example, Wilcken et al. (2) were the first to report the occurrence of AFLP and HELLP syndrome in six of six pregnancies where the fetus was LCHAD deficient. Subsequently, Tyni et al. (3) retrospectively examined 63 pregnancies resulting in LCHAD-deficient newborns, and reported a statistically significant correlation with the occurrence of either AFLP or HELLP syndrome in the mother. Ibdah et al. (4) found that 15 of $19(79 \%)$ mothers carrying an LCHADdeficient fetus developed either AFLP or HELLP syndrome. Notably, in all testable pregnancies, if the fetus was normal or 
a simple heterozygote, no maternal complications were noted. These reports emphasized the potentially sizable contribution of fetus-to-maternal pregnancy-related complications.

LCHAD activity in humans is restricted to the $\alpha$-subunit of the mitochondrial trifunctional protein, and IJlst et al. (5) were the first to demonstrate that a common $\mathrm{G}$ to $\mathrm{C}$ mutation at position 1528 (G1528C) of the $\alpha$-subunit directly results in LCHAD deficiency. Subsequently, Sims et al. (6) extended the study of LCHAD deficiency to three infants with biallelic mutations in the LCHAD coding region. Two of three children were homozygous for the G1528C mutation, whereas one fetus was a compound heterozygote, harboring one allele with the G1528C mutation. The mothers of the affected infants developed AFLP or HELLP syndrome in the course of their pregnancy, and in all three cases, the $\mathrm{G} 1528 \mathrm{C}$ mutation was inherited from the mother. These investigators then examined 16 additional children with LCHAD deficiency and found the presence of a $\mathrm{G} 1528 \mathrm{C}$ allele in $100 \%$ of cases, either as a homozygous or compound heterozygote mutation (4). Importantly, in as many as 13 of $19(68 \%)$ cases, the G1528C allele was inherited from the mother.

The major limitation of existing studies is that all have examined LCHAD mutations in the mother by using enzyme deficiency in the fetus as an inclusion criterion $(2-4,6)$. However, there are scant studies examining the prevalence of LCHAD mutations in an "unselected" population of obstetric patients with AFLP, irrespective of the status of LCHAD in the fetus. Given that the incidence of AFLP in the obstetric population is at most 1 in 7,000 (7), and the incidence of LCHAD deficiency in the general population is approximately 1 in 70,000 (1), it is intuitive that the previous study designs would overestimate the frequency of association between the two entities. Therefore, we decided to examine for the presence of the common G1528C LCHAD mutation retrospectively in a group of obstetric patients who developed AFLP during pregnancy. To avoid a selection bias, the LCHAD status of the fetus was not a criterion for inclusion in the study.

\section{MATERIALS AND METHODS}

The clinical records of the Department of Obstetrics and Gynecology at University of Texas Southwestern Medical Center were reviewed to retrieve patients with AFLP documented during pregnancy. The diagnosis of AFLP was made on the basis of clinical and laboratory criteria as reported by Castro et al. (7). A retrospective review of the archives of the Department of Pathology was then performed to select the subset of cases that had paraffin-embedded material available from surgical procedures or biopsies. Of all patients who were diagnosed with AFLP in the period between 1985 and 1997, 10 had adequate archival tissue amenable to DNA analysis, including 3 with peripartum liver biopsies. The mean maternal age at diagnosis was 30.9 y (range 16-40 y). The mean gestational age at diagnosis of AFLP was $36.6 \mathrm{wk}$ (range 31.1-40.7 wk). The study series consisted of six Hispanic patients, three African Americans, and one Caucasian. The studies were carried out with the approval of the Institutional Review Board of the University of Texas Southwestern Medical Center.
The sources of constitutional maternal DNA in the 10 cases were as follows: liver ( 3 cases), placenta ( 3 cases), fallopian tube/adnexa ( 3 cases), and perineal cyst ( 1 case). Because our objective was to examine DNA exclusively from maternal tissues, laser capture microdissection was performed using the Arcturus PixCell II instrument (Arcturus Engineering, Mountain View, CA, U.S.A.) (8) to exclude contamination by fetal cells in the three placentas. This involved selective isolation of the maternal decidual cells from the decidua basalis and parietalis, leaving the placental membranes and villi intact. Despite the absence of fetal cells, laser capture microscopy was performed in the other seven cases as well, to maintain a uniform protocol for cell numbers and DNA extraction. Briefly, multiple serial $5-\mu \mathrm{m}$ sections were cut on uncharged glass slides, stained with hematoxylin and eosin, and one level was coverslipped for use as a visual guide for microdissection. Approximately 1000 cells were microdissected from the remaining serial sections, and DNA was extracted using $5 \mu \mathrm{m}$ of proteinase $\mathrm{K}$ for every 100 cells, as previously described (9). The presence of genomic DNA that could by amplified by PCR in each case was verified using a second gene, medium-chain acyl-CoA dehydrogenase.

Current existing primers for rapid screening of the G1528C mutation in the $\alpha$-subunit produce an amplicon size of $640 \mathrm{bp}$ (10). Because DNA extracted from archival tissues is usually not amenable to successful amplification beyond $250 \mathrm{bp}$ (11), we modified the 3 -antisense primer sequence to result in an amplicon of $128 \mathrm{bp}$, which includes a previously described Pst 1 restriction site for restriction fragment length polymorphism analysis (10). The trifunctional protein $\alpha$-subunit shows $93 \%$ homology with a pseudogene (12). To circumvent the possibility of amplifying the pseudogene, two safeguards were introduced in the primer design: the 5 '-sense primer on exon 15 included flanking intronic sequences (10), and the 3 '-antisense primer was designed to have a 2-bp mismatch with the pseudogene (12). The oligonucleotide sequences were as follows:

Sense: 5'-CCC TTG CCA GGT GAT TGG C-3'

Antisense: 5'-CCT GCT TGA GAC CAA CTG CT-3'

Ten microliters of proteinase $\mathrm{K}$-digested genomic DNA was used in a total reaction mixture of $50 \mu \mathrm{L}$ for each PCR, with the following reaction conditions: initial denaturation at $94^{\circ} \mathrm{C}$ for $5 \mathrm{~min}$, followed by 40 cycles of denaturation at $94^{\circ} \mathrm{C}$ $(30 \mathrm{~s})$, annealing at $55^{\circ} \mathrm{C}(30 \mathrm{~s})$, and extension at $72^{\circ} \mathrm{C}(30 \mathrm{~s})$. The final extension was for $5 \mathrm{~min}$. Genomic DNA from a LCHAD-heterozygous mutant positive control was concurrently run with the test samples. Ten microliters of the final amplified product was digested with $P$ st 1 at $37^{\circ} \mathrm{C}$ for $120 \mathrm{~min}$. Pre- and postdigestion samples were subjected to electrophoresis on a $10 \%$ polyacrylamide gel, and visualized by ethidium bromide staining. Undigested PCR-amplified product from two representative cases was sequenced on the ABI 377 automated sequencer (Applied Biosystems, Foster City, CA) as a quality control for primer specificity.

\section{RESULTS}

Successful DNA amplification was possible in all cases using both medium-chain acyl-CoA dehydrogenase and 
LCHAD primers. Pre- and post-PstI digestion products showed the presence of the 128-bp amplified LCHAD product in all samples, including the LCHAD-heterozygous positive control. Pst I digestion of the heterozygous control yielded three bands: the 128-bp product of the normal allele and two smaller 71and 57-bp fragments from the mutant allele. The 10 test samples yielded only the 128 -bp product after restriction digestion (Fig. 1). The sequence of the amplified PCR product was verified in two representative samples and the heterozygous mutant LCHAD control as the genomic sequence of the trifunctional gene $\alpha$-subunit (data not shown).

\section{DISCUSSION}

Pregnant women carrying fetuses with LCHAD deficiency are at risk for developing severe pregnancy-related complications of AFLP and/or the HELLP syndrome $(4,6,13,14)$. The first description of the possibility of an association was proposed in 1991, when Schoeman et al. (15) reported two episodes of AFLP in a mother whose pregnancies resulted in infants with suspected biochemical defects. Although the specific defect was not known, a disorder of fatty acid oxidation was suspected on the basis of the characteristic clinical phenotype. Subsequent reports identified the
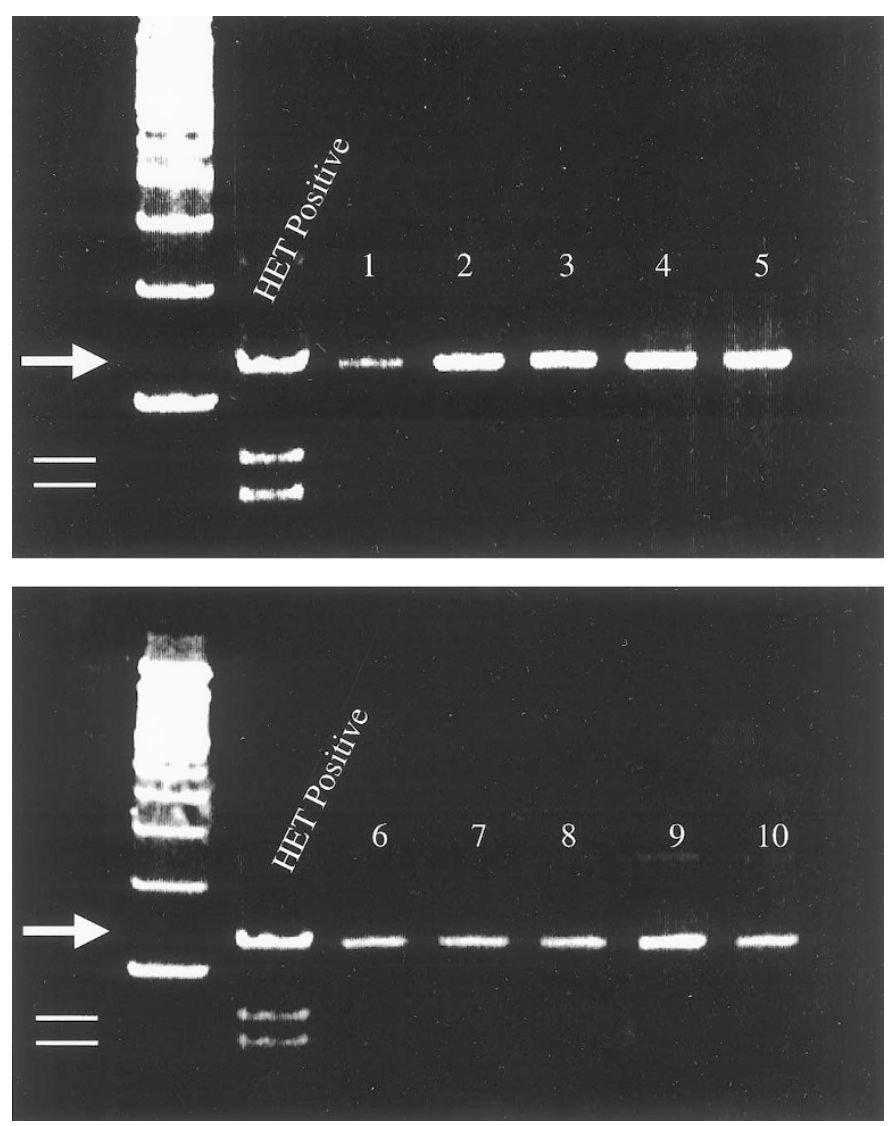

Figure 1. Electrophoresis of samples from patients with AFLP, after PCR amplification and Pst I digestion, to determine the presence of G1528C mutation. The LCHAD-deficient heterozygote positive (HET positive) control shows the expected pattern of banding: the wild-type allele produces a 128-bp product, and the mutant allele, containing the Pst I restriction site, produces two smaller bands ( 71 and $58 \mathrm{bp}$ ). In contrast, all 10 patient samples only produce the 128-bp product, consistent with absence of G1528C mutation on both alleles. fetal metabolic defect as LCHAD deficiency and described a clear association between fetal deficiency for the protein and the development of either AFLP or HELLP syndrome during pregnancy (2, $4,6,13,14,16)$. Therefore, it followed that the fetus would be homozygous or a compound heterozygote for mutations producing LCHAD deficiency. The "common" LCHAD mutation is replacement of a guanine by a cytosine residue at nucleotide 1528 of the LCHAD gene (10), and, indeed, this mutation has been found, at least in one copy, in almost all LCHAD-deficient infants from pregnancies complicated by $\operatorname{AFLP}(4,6,14)$.

The largest and most recent study describing an association of AFLP/HELLP syndrome and LCHAD deficiency evaluated 24 mother and infant pairs using DNA analysis for all 20 exons of the LCHAD gene (4). Of the 24 infants evaluated, 19 had isolated LCHAD deficiency, and in $15(79 \%)$, there was a history of a pregnancy complicated by AFLP. In $100 \%$ of mothers who developed AFLP, the fetal metabolic defect was the result of either a homozygous G1528C mutation, or compound heterozygous mutation, which included the G1528C mutation on one of the alleles. There were five infants with complete trifunctional protein deficiency, but not the G1528C mutation, who were delivered to mothers with uncomplicated pregnancies, indicating that the presence of G1528C allele may be a prerequisite in the LCHAD-deficient fetus for development of AFLP in the mother.

Despite the strong association of fetal LCHAD deficiency and AFLP, our study failed to identify the common LCHAD mutation in tissue from 10 patients with either biopsy-proven or clinically diagnosed AFLP. Although the literature might suggest that the LCHAD mutation is common in pregnancies complicated by AFLP, there is likely a significant selection bias in the cases that have been reported as a result of ascertainment through an affected infant. Our results are similar to those reported by Mansouri et al. (17), who did not find the common LCHAD mutation in any of 14 French women with AFLP. In a larger study based on 113 Dutch women with the HELLP syndrome, den Boer et al. (18) detected the G1528C mutation in only one patient.

There are many potential reasons for not detecting the G1528C mutation in the tissue from mothers with AFLP. Although the G1528C mutation has been detected on at least one allele in all infants described from families with LCHAD deficiency and AFLP, the infants frequently carry a different mutation on the other affected allele. The mother may therefore not carry the G1528C mutation, but a different mutation. It is noteworthy, however, that in over two thirds of compound heterozygotes with LCHAD deficiency, it is the mother who transmits the G1528C allele (4). Despite this caveat, the G1528C mutation may be inherited from the father, and tissue from the fathers was not available to us for study. Secondly, our population in Dallas is strongly Hispanic, and the LCHAD mutation may not be as prevalent in the Hispanic population compared with people of European descent, where the majority of the cases of the LCHAD mutation and AFLP have been described. In addition, and probably of major importance, our population was ascertained through maternal presentation of AFLP and not from an infant carrying the metabolic defect. Mansouri et al. (17) were the first to suggest a strong selection 
bias of cases previously reported, and our results are in concert with theirs. Each of these small studies or case reports was ascertained through a child affected with LCHAD deficiency, so the prevalence of the mutation would stand to be higher than in the population as a whole. Clearly, fetal LCHAD deficiency is strongly associated with AFLP in families with the genetic defect, but the association with AFLP in populations without a family history is less striking. The recent report of maternal liver disease arising in the backdrop of fetal hepatic carnitine palmitoyltransferase I deficiency reinforces that the metabolic basis of AFLP is more heterogeneous than previously believed (19).

Despite the fact that we could not identify an LCHAD mutation in any of our samples, we did devise a protocol for identifying the mutation in formalin-fixed microdissected samples, making retrospective evaluation possible for the LCHAD gene in rare diseases such as AFLP. We initially attempted to use the primer sequences identified, which span the mutation and give an amplified product of $640 \mathrm{bp}$ (10). Unfortunately, we did not obtain DNA of a sufficient length to amplify this relatively long product from our samples because DNA fragmentation, which occurs with tissue fixation (11). Therefore, we designed our primers to amplify a short DNA fragment of 128 bp that included the G1528C mutation. Because amplifying the pseudogene is of potential concern, we included safeguards in our primer design (see "Materials and Methods"). Additionally, we confirmed that the amplified sequence was the LCHAD gene, and not the pseudogene, by sequence analysis of the amplified product.

We report the absence of the common LCHAD mutation in an unselected series of patients with AFLP. In concert with the study by den Boer et al. (18), it appears that heterozygous LCHAD mutations are infrequent in both AFLP and HELLP syndrome occurring in the general obstetric population. These findings, however, do not negate the known association of maternal LCHAD mutation and AFLP in the small subset of families with LCHAD deficiency in the offspring. Abnormal LCHAD function may represent only one of a variety of metabolic disorders that results in the clinical phenotype of fatty metamorphosis of the liver in the pregnant woman.

\section{REFERENCES}

1. Strauss AW, Bennett MJ, Rinaldo P, Sims HF, O’Brien LK, Gibson B, Ibdah J 1999 Inherited long-chain 3-hydroxyacyl-CoA dehydrogenase deficiency and a fetal- maternal interaction cause maternal liver disease and other pregnancy complications. Semin Perinatol 23:100-112

2. Wilcken B, Leung KC, Hammond J, Kamath R, Leonard JV 1993 Pregnancy and fetal long-chain 3-hydroxyacyl coenzyme A dehydrogenase deficiency. Lancet 341:407408

3. Tyni T, Ekholm E, Pihko H 1998 Pregnancy complications are frequent in long-chain 3-hydroxyacyl-coenzyme A dehydrogenase deficiency. Am J Obstet Gynecol 178:603-608

4. Ibdah JA, Bennett MJ, Rinaldo P, Zhao Y, Gibson B, Sims HF, Strauss AW 1999 A fetal fatty-acid oxidation disorder as a cause of liver disease in pregnant women. N Engl J Med 340:1723-1731

5. IJlst L, Wanders RJ, Ushikubo S, Kamijo T, Hashimoto T 1994 Molecular basis of long-chain 3-hydroxyacyl-CoA dehydrogenase deficiency: identification of the major disease-causing mutation in the alpha-subunit of the mitochondrial trifunctional protein. Biochim Biophys Acta 1215:347-350

6. Sims HF, Brackett JC, Powell CK, Treem WR, Hale DE, Bennett MJ, Gibson B, Shapiro S, Strauss AW 1995 The molecular basis of pediatric long chain 3-hydroxyacyl-CoA dehydrogenase deficiency associated with maternal acute fatty liver of pregnancy. Proc Natl Acad Sci U S A 92:841-845

7. Castro MA, Fassett MJ, Reynolds TB, Shaw KJ, Goodwin TM 1999 Reversible peripartum liver failure: a new perspective on the diagnosis, treatment, and cause of acute fatty liver of pregnancy, based on 28 consecutive cases. Am J Obstet Gynecol $181: 389-395$

8. Bonner RF, Emmert-Buck M, Cole K, Pohida T, Chuaqui R, Goldstein S, Liotta LA 1997 Laser capture microdissection: molecular analysis of tissue. Science 278:14811483

9. Wistuba II, Behrens C, Milchgrub S, Virmani A, Jagirdar J, Thomas B, Ioachim HL, Litzky LA, Brambilla E, Minna JD, Gazdar AF 1998 Comparison of molecular changes in lung cancers in HIV-positive and HIV-indeterminate subjects. JAMA 279:1554-1559

10. IJlst L, Ruiter JP, Hoovers JM, Jakobs ME, Wanders RJ 1996 Common missense mutation G1528C in long-chain 3-hydroxyacyl-CoA dehydrogenase deficiency. Characterization and expression of the mutant protein, mutation analysis on genomic DNA and chromosomal localization of the mitochondrial trifunctional protein alpha subunit gene. J Clin Invest 98:1028-1033

11. Maitra A, Wistuba II, Virmani AK, Sakaguchi M, Park I, Stucky A, Milchgrub S, Gibbons D, Minna JD, Gazdar AF 1999 Enrichment of epithelial cells for molecular studies. Nat Med 5:459-463

12. Zhang QX, Baldwin GS 1994 Structures of the human cDNA and gene encoding the $78 \mathrm{kDa}$ gastrin-binding protein and of a related pseudogene. Biochim Biophys Acta 1219:567-575

13. Treem WR, Rinaldo P, Hale DE, Stanley CA, Millington DS, Hyams JS, Jackson S, Turnbull DM 1994 Acute fatty liver of pregnancy and long-chain 3-hydroxyacylcoenzyme A dehydrogenase deficiency. Hepatology 19:339-345

14. Isaacs Jr JD, Sims HF, Powell CK, Bennett MJ, Hale DE, Treem WR, Strauss AW 1996 Maternal acute fatty liver of pregnancy associated with fetal trifunctional protein deficiency: molecular characterization of a novel maternal mutant allele. Pediatr Res 40:393-398

15. Schoeman MN, Batey RG, Wilcken B 1991 Recurrent acute fatty liver of pregnancy associated with a fatty-acid oxidation defect in the offspring. Gastroenterology 100:544-548

16. Treem WR, Shoup ME, Hale DE, Bennett MJ, Rinaldo P, Millington DS, Stanley CA, Riely CA, Hyams JS 1996 Acute fatty liver of pregnancy, hemolysis, elevated liver enzymes, and low platelets syndrome, and long chain 3-hydroxyacyl-coenzyme A dehydrogenase deficiency. Am J Gastroenterol 91:2293-2300

17. Mansouri A, Fromenty B, Durand F, Degott C, Bernuau J, Pessayre D 1996 Assessment of the prevalence of genetic metabolic defects in acute fatty liver of pregnancy. J Hepatol 25:781

18. den Boer ME, IJlst L, Wijburg FA, Oostheim W, van Werkhoven MA, van Pampus MG, Heymans HS, Wanders RJ 2000 Heterozygosity for the common LCHAD mutation $(1528 \mathrm{G}>\mathrm{C})$ is not a major cause of HELLP syndrome and the prevalence of the mutation in the Dutch population is low. Pediatr Res 48:151-154

19. Innes AM, Seargeant LE, Balachandra K, Roe CR, Wanders RJ, Ruiter JP, Casiro O, Grewar DA, Greenberg CR 2000 Hepatic carnitine palmitoyltransferase I deficiency presenting as maternal illness in pregnancy. Pediatr Res 47:43-45 